\title{
Pionic decay of $\Lambda$ Hypernuclei in a Continuum Shell Model
}

\author{
C. Albertus, J.E. Amaro and J. Nieves \\ Departamento de Física Moderna, Universidad de Granada, E-18071 Granada, Spain.
}

\begin{abstract}
We evaluate pionic decay widths of $\Lambda$ hypernuclei using a shell model for, both the nuclear bound and the continuum nucleon wave functions in the final state, and distorted waves for the outgoing pion. An excellent agreement with the recent KEK measurement of $\pi^{-}$-decay widths of ${ }_{\Lambda}^{12} \mathrm{C}$ and ${ }_{\Lambda}^{28} \mathrm{Si}$ is found. Besides, results for ${ }_{\Lambda}^{56} \mathrm{Fe}$ are consistent with the existing upper bound.
\end{abstract}

PACS: 21.80+a, 23.90.+w, 25.80.Pw,13.30.-a, 23.20.Js, 21.60.Cs

Keywords: $\Lambda$ hypernuclei, weak decay, mesonic decay, continuum shell model . 


\section{Introduction}

The pionic decay of $\Lambda$ hypernuclei has received attention in the past both from the experimental [1]-[7] and theoretical [8]-[22] points of view (for a recent review see Ref. [23]). It is well known that the mesonic decay is largely suppressed by Pauli blocking, although the consideration of proper pion distorted waves $[13,16,20]$ weakens the effect of the Pauli blocking considerably. On the other hand, it was also pointed out in Ref. [16] the relevance of the use of a correct energy balance in the decay. Two theoretical frameworks have been traditionally used to describe this process: Polarization Propagator Method (PPM) [12, 18, 21], supplemented by quantum field theory functional techniques [22, 23], and the Wave Function Method (WFM) [13]-[17]. In the PPM, the calculation of the pionic widths is performed in nuclear matter and the results are translated to finite nuclei by means of the Local Density Approximation (LDA). Both methods PPM and WFM turn out to be related in a semiclassical limit [19], being the WFM more reliable than the $\mathrm{PPM}+\mathrm{LDA}$, since the mesonic decay channel is quite sensitive to the details of the shell structure of the hypernuclei, specially for light systems.

In Ref. [16] a simple Woods-Saxon (WS) shell model was employed, with a central potential of constant depth for several nuclei. This global potential did not include spinorbit term and only the radius of the WS well depended on the specific nuclei $\left(\propto A^{\frac{1}{3}}\right)$. The resulting binding energies of the shells were globally shifted, by hand, to reproduce the ground state masses of the involved nuclei. Besides, the continuum contribution to the decay was estimated by discretizing the positive nucleon energy levels by means of an infinite barrier placed at distances of about $20 \mathrm{fm}$. Despite all these crude approximations, the model led to predictions for the mesonic decay widths of ${ }_{\Lambda}^{12} \mathrm{C}$ which were compatible, within errors, with the available experimental measurements at that time, for both $\pi^{0}$ and $\pi^{-}$decay channels. Furthermore, the model of Ref. [16] provided an overall description of medium and heavy hypernuclei mesonic decay, which is still nowadays accepted as one of the most reliable theoretical estimates [23].

Very recently, precise measurements of the $\pi^{-}$mesonic decay of ${ }_{\Lambda}^{12} \mathrm{C}$ and ${ }_{\Lambda}^{28}$ Si have been obtained at KEK, and an upper bound for ${ }_{\Lambda}^{56} \mathrm{Fe}$ has also been given [7]. The purpose of this paper is to update the model of Ref. [16], improving the nuclear structure description

of the process, by using: i) more realistic potentials for each one of the nuclei by fitting the parameters of a WS well, with spin-orbit forces, to the ground state and also to some of the discrete final state energies of the nuclei involved in the decay and ii) a Continuum Shell Model (CSM) to describe the positive energy tail of the mesonic decay width. In this way we replace the discrete sum used in Ref. [16] by the appropriate integral over the continuum states. This correct treatment of the continuum contribution is important, since, as we shall see, for $\pi^{-}{ }^{28}{ }_{\Lambda} \mathrm{Si}$ decay it amounts about $50 \%$ of the total measured mesonic width, in contrast to the case of the hypernuclei studied in Refs. [13] and [16], for which the continuum contribution was much smaller.

Since, as mentioned above, the use of pion distorted waves turns out to be crucial [16], [20], the improved treatment of the nuclear structure allows us to use the new and precise measurements performed at KEK, as a further test of the quality of the pion-nucleus 
dynamics used in Ref. [16] and developed in the works of Refs. [24] and [25]. A deep and detailed knowledge of the pion dynamics inside of a nuclear medium has become a topic of renewed interest to explore possible partial chiral restoration in the medium [26]-[28].

The paper is organized as follows: In Sect. 2 the needed formulae to compute the pionic decay width are given, both for the discrete and continuum contributions. Details on the nuclear CSM used in this work are discussed in Sect. 3, where the energy reaction balance is also studied. Finally in Sect. 4 we present the results of this work and our main conclusions.

\section{Theoretical Description of the Pionic Decay.}

In this work, we compute the mesonic decay width as the sum of the contributions of the following processes ${ }^{1}$

$$
\begin{aligned}
& { }_{\Lambda}^{A} \mathrm{Z} \rightarrow\left({ }^{A} \mathrm{Z}\right)_{d}+\pi^{0}, \\
& { }_{\Lambda}^{A} \mathrm{Z} \rightarrow\left({ }^{A}(\mathrm{Z}+1)\right)_{d}+\pi^{-}, \\
& { }_{\Lambda}^{A} \mathrm{Z} \rightarrow\left({ }^{A-1} \mathrm{Z}\right)_{g s}+n+\pi^{0}, \\
& { }_{\Lambda}^{A} \mathrm{Z} \rightarrow\left({ }^{A-1} \mathrm{Z}\right)_{g s}+p+\pi^{-},
\end{aligned}
$$

where $d$ denotes the ground or discrete excited states of the final nucleus. As we shall see, we evaluate the processes of Eqs.(1), (2) by putting the outgoing nucleon, coming from the weak $\Lambda$ decay, in an unoccupied bound shell of the daughter nucleus. On the other hand, in Eqs. (3) and (4) gs means that the daughter nucleus is left on its ground state. In the two last reactions the outgoing nucleon, coming from the weak $\Lambda$ decay, goes to the continuum (positive energy) and we denote that contribution by $\Gamma_{c}$, while the first two reactions give what we call discrete contribution $\Gamma_{d}$. Thus we split the pionic width into two contributions, $\Gamma=\Gamma_{c}+\Gamma_{d}$. Experimentally, what can be observed are the inclusive processes

$$
\begin{aligned}
& { }_{\Lambda}^{A} \mathrm{Z} \rightarrow X+\pi^{0}, \\
& { }_{\Lambda}^{A} \mathrm{Z} \rightarrow X+\pi^{-} .
\end{aligned}
$$

The main contribution to these processes is given by the exclusive ones shown in Eqs. (14).

The pionic decay is produced by a baryonic one-body operator

$$
\delta \widetilde{H}_{\Lambda \pi N}^{\lambda}=-G m_{\pi}^{2}\left\{S-\frac{P}{m_{\pi}} \vec{\sigma} \cdot \vec{q}_{c m}\right\} \tau^{\lambda},
$$

where $\left(G m_{\pi}^{2}\right)^{2} / 8 \pi=1.945 \times 10^{-15}$, the constants $S$ and $P$ are equal to 1.06 and 0.527 respectively and $m_{\pi}$ is the pion mass $\left(139.57\right.$ or $134.98 \mathrm{MeV}$ for $\pi^{-}$or $\pi^{0}$ ), $\vec{q}_{c m}$ is the

\footnotetext{
${ }^{1}$ We use the notation ${ }_{\Lambda}^{A} Z$, to denote a nuclear core ${ }^{A-1} Z$ and one bound $\Lambda$ particle.
} 
momentum of the outgoing pion in the $\Lambda$ rest frame, and the Pauli matrices $\vec{\sigma}$ and $\tau^{\lambda}$, where $\lambda$ is a cartesian isospin index which will be contracted to the pion field, act on the spin and isospin Hilbert spaces respectively. Taking the $\Lambda$ isospin wave function as that of a neutron, the $\tau^{\lambda}$ operator in Eq. (7) implements the extreme $\Delta T=1 / 2$ rule, which leads to a rate of $\Lambda \rightarrow \pi^{-} p$ twice as large as that of $\Lambda \rightarrow \pi^{0} n$.

The free space $\Lambda$ decay width is readily evaluated and leads for proton or neutron decay to

$$
\begin{aligned}
\Gamma_{\text {free }}^{(\alpha)} & =C^{(\alpha)} \frac{\left(G m_{\pi}^{2}\right)^{2}}{4 \pi} \frac{M q_{c m}}{M_{\Lambda}}\left\{S^{2}+\left(\frac{P}{m_{\pi}}\right)^{2} q_{c m}^{2}\right\}, \quad \alpha=p, n \\
q_{c m} & =\frac{\lambda^{1 / 2}\left(M_{\Lambda}^{2}, M^{2}, m_{\pi}^{2}\right)}{2 M_{\Lambda}}, \\
\lambda(x, y, z) & =x^{2}+y^{2}+z^{2}-2 x y-2 x z-2 y z,
\end{aligned}
$$

where $\alpha$ indicates neutron or proton or equivalently $\pi^{0}$ or $\pi^{-}$decay, $C^{(p)}=4$ and $C^{(n)}=2$ are isospin coefficients, and $M(938.27$ or $939.57 \mathrm{MeV}$ for $p$ or $n)$ and $M_{\Lambda}(1115.68 \mathrm{MeV})$ are the nucleon and $\Lambda$ masses respectively. The total free space hyperon $\Lambda$ width, $\Gamma_{\Lambda}$, is given by the sum of proton $\left(\pi^{-}\right)$and neutron $\left(\pi^{0}\right)$ contributions,

$$
\Gamma_{\Lambda}=\Gamma_{\text {free }}^{(p)}+\Gamma_{\text {free }}^{(n)}
$$

In the case of a bound hypernucleus, assuming that the hyperon $\Lambda$ is in the $1 s_{\frac{1}{2}}$ shell and a closed shell structure for the underlying nuclear system, ${ }^{A-1} \mathrm{Z}$, the width for any of the processes of Eqs. (1-4) is given by ${ }^{2}$

$$
\begin{aligned}
\Gamma^{(\alpha)} & =C^{(\alpha)} \sum_{N=n l j m>F} \frac{1}{2} \sum_{m_{s_{\Lambda}}} \int \frac{d^{3} q}{(2 \pi)^{3}} \frac{1}{2 \omega(q)} 2 \pi \delta\left(E_{\Lambda}-\omega(q)-E_{N}\right) \\
& \times\left(G m_{\pi}^{2}\right)^{2}\left|\left\langle\Lambda, m_{s_{\Lambda}}\left|\left[S-\frac{P}{m_{\pi}} \vec{\sigma} \cdot \vec{\nabla}_{\pi}\right]\right| n l j m ; \widetilde{\varphi}_{\pi}^{(\alpha)}(\vec{q})^{*}\right\rangle\right|^{2}
\end{aligned}
$$

where $n l j m$ and $E_{N}$ stands for the quantum numbers and relativistic energy of the outgoing nucleon in the $n l j$ shell, $\left|\Lambda, m_{s_{\Lambda}}\right\rangle$ denotes the $\Lambda$ wave function with angular momentum third component $m_{s_{\Lambda}}, E_{\Lambda}$ the $\Lambda$ energy, including its mass, $\omega(q)$ the pion energy, and the sum over $N$ runs over the unoccupied nuclear orbitals $(n, l, j, m)$. In Eq. (12) the sums over $N$ are over proton or neutron orbitals according to $\alpha$. The pion wave function $\left(\widetilde{\varphi}_{\pi}^{(\alpha)}(\vec{q}, x)^{*}\right)$ as a block corresponds to an incoming solution of the Klein Gordon equation,

$$
\left[-\vec{\nabla}^{2}+m_{\pi}^{2}+2 \omega(q) V_{\mathrm{opt}}(\vec{x})\right] \widetilde{\varphi}_{\pi}^{(\alpha)}(\vec{q}, \vec{x})^{*}=\left(\omega(q)-V_{C}(\vec{x})\right)^{2} \widetilde{\varphi}_{\pi}^{(\alpha)}(\vec{q}, \vec{x})^{*},
$$

with $V_{C}(\vec{x})$ the Coulomb potential created by the nucleus considering finite size and vacuum polarization effects, for $\pi^{-}$and zero for $\pi^{0}$, and $V_{\text {opt }}(\vec{x})$ the optical potential which

\footnotetext{
${ }^{2}$ The following expressions are valid for the discrete contribution (Eqs. (1) and (2)) to the decay width. The needed modifications to compute the continuum part (Eqs. (3) and (4)) are discussed after Eq. (24).
} 
describes the $\pi$-nucleus interaction. This potential has been developed microscopically and it is exposed in detail in Refs. [24, 25]. It contains the ordinary lowest order optical potential pieces constructed from the $s$ - and $p$-wave $\pi N$ amplitudes. In addition second order terms in both $s$ - and $p$-waves, responsible for pion absorption, are also considered. Standard corrections, as second-order Pauli re-scattering term, ATT term, Lorentz-Lorenz effect and long and short range nuclear correlations, are also taken into account. This theoretical potential reproduces fairly well the data of pionic atoms (binding energies and strong absorption widths) [24] and low energy $\pi$-nucleus scattering [25].

After a little Racah algebra, one gets for the $\Lambda$ decay width inside of a nucleus

$$
\begin{aligned}
\Gamma^{(\alpha)} & =\sum_{N=n l j>F} \Gamma_{N}^{(\alpha)} \\
\Gamma_{N}^{(\alpha)} & =\frac{C^{(\alpha)}}{4 \pi}\left(G m_{\pi}^{2}\right)^{2} \frac{q_{N}}{1+\omega\left(q_{N}\right) / E_{A}}\left[S^{2} S_{N}^{(s)}\left(q_{N}\right)+\left(\frac{P}{m_{\pi}}\right)^{2} \vec{q}_{N}^{2} S_{N}^{(p)}\left(q_{N}\right)\right]
\end{aligned}
$$

with

$$
q_{N}=\left(\left(E_{\Lambda}-E_{N}\right)^{2}-m_{\pi}^{2}\right)^{1 / 2}
$$

Note that $E_{N}, q_{N}$ and the integrals $S_{N}^{(s)}$ and $S_{N}^{(p)}$, defined below, depend on the isospin $\alpha$. We have implemented the recoil factor $\left(1+\omega / E_{A}\right)^{-1}$, being $E_{A}$ the energy (including the mass) of the daughter nucleus, because most of the decay corresponds to nucleons in nuclear bound excited states, and as a consequence the nucleus of mass $M_{A}$ recoils as a whole. $S_{N}^{(s)}\left(q_{N}\right)$ and $S_{N}^{(p)}\left(q_{N}\right)$ are the $s$-wave and $p$-wave contributions given by

$$
\begin{aligned}
S_{n l j}^{(s)}\left(q_{N}\right) & =\frac{2 j+1}{2}\left|I_{n l j}\left(q_{N}\right)\right|^{2} \\
S_{n l j}^{(p)}\left(q_{N}\right) & =\frac{1}{{\overrightarrow{q_{N}}}^{2}}\left\{l\left|M_{n l j}\left(q_{N}\right)\right|^{2} \delta_{j, l-1 / 2}+(l+1)\left|N_{n l j}\left(q_{N}\right)\right|^{2} \delta_{j, l+1 / 2}\right\}
\end{aligned}
$$

with

$$
\begin{aligned}
I_{n l j}\left(q_{N}\right) & =\int_{0}^{\infty} r^{2} d r R_{1 s}^{(\Lambda)}(r) R_{l}^{(\pi)}\left(q_{N} ; r\right) R_{n l j}(r) \\
M_{n l j}\left(q_{N}\right) & =\int_{0}^{\infty} r^{2} d r R_{1 s}^{(\Lambda)}(r)\left(\frac{d R_{l-1}^{(\pi)}\left(q_{N} ; r\right)}{d r}-(l-1) \frac{R_{l-1}^{(\pi)}\left(q_{N} ; r\right)}{r}\right) R_{n l j}(r) \\
N_{n l j}\left(q_{N}\right) & =\int_{0}^{\infty} r^{2} d r R_{1 s}^{(\Lambda)}(r)\left(\frac{d R_{l+1}^{(\pi)}\left(q_{N} ; r\right)}{d r}+(l+2) \frac{R_{l+1}^{(\pi)}\left(q_{N} ; r\right)}{r}\right) R_{n l j}(r) .
\end{aligned}
$$

Here $R_{l}^{(\pi)}\left(q_{N} ; r\right)$ are the radial wave functions of the pion for each partial wave, regular in the origin and with the asymptotic behavior

$$
R_{l}^{(\pi)}(q ; r)_{r \rightarrow \infty} \simeq e^{i \delta_{l}} \frac{1}{q r} \sin \left(q r-l \frac{\pi}{2}+\delta_{l}\right) \text { for } \pi^{0}
$$




$$
R_{l}^{(\pi)}(q ; r)_{r \rightarrow \infty} \simeq e^{i\left(\delta_{l}+\sigma_{l}\right)} \frac{1}{q r} \sin \left(q r-l \frac{\pi}{2}+\sigma_{l}+\delta_{l}-\eta \log 2 q r\right) \text { for } \pi^{-}
$$

with $\eta$ and $\sigma_{l}$ the Coulomb parameter and phase shift defined as in Ref. [29] and $\delta_{l}$ the complex (to take into account inelasticities) phase shifts obtained from the numerical solution of the Klein Gordon equation. Finally, $R_{1 s}^{(\Lambda)}$ and $R_{n l j}(r)$ are the $\Lambda$ and nucleon bound radial wave functions, normalized as usual

$$
\int_{0}^{+\infty} d r r^{2}|R(r)|^{2}=1
$$

The $\Lambda$ wave function in the initial hypernucleus, $R^{(\Lambda)}$, is obtained from a WS potential [33] to account for the mean $\Lambda$-nuclear core interaction, with parameters compiled in Table 1. The nucleon dynamics will be studied in detail in Sect. 3.

Some of the nuclei which we use are not closed shell nuclei. In this case the nucleons from the $\Lambda$ decay can fill up $n_{h}$ empty states in a $n, l, j$ shell. We take that into account by multiplying $S_{N}^{(s)}$ and $S_{N}^{(p)}$ by $n_{h} /(2 j+1)$.

\begin{tabular}{ccccc}
\hline Hypernucleus & $V_{0}^{\Lambda}[\mathrm{MeV}]$ & $R^{\Lambda}[\mathrm{fm}]$ & $a^{\Lambda}[\mathrm{fm}]$ & $B_{\Lambda}[\mathrm{MeV}]$ \\
\hline${ }_{\Lambda}^{12} \mathrm{C}$ & 31.1 & 2.45 & 0.60 & $-10.8[30]$ \\
${ }_{\Lambda}^{28} \mathrm{Si}$ & 30.1 & 3.30 & 0.60 & $-16.6[31]$ \\
${ }_{\Lambda}^{56} \mathrm{Fe}$ & 30.3 & 4.18 & 0.60 & $-21.0[11]$ \\
\hline
\end{tabular}

Table 1: Lambda hyperon WS parameters and ground state $\left(1 s_{\frac{1}{2}}\right)$ binding energies $\left(B_{\Lambda}\right)$. The total $\Lambda$ energy, $E_{\Lambda}$ is given by $M_{\Lambda}+B_{\Lambda}$.

The above equations (12)-(24) can be readily used to compute the discrete contribution to the pionic decay width, but when the outgoing nucleon coming from the weak $\Lambda$ decay goes to the continuum (positive energy), one should replace the sum $\sum_{N=n l j>F}$ in Eq. (14) by a sum over multipoles, plus an integral over the nucleon energies, i.e.,

$$
\begin{aligned}
\sum_{N=n l j>F} & \rightarrow \sum_{l j} \int_{M}^{E_{\max }} d E \frac{2 M p}{\pi} \\
R_{n l j}(r) & \rightarrow R_{l j}(p ; r)
\end{aligned}
$$

with $E$ and $p=\sqrt{E^{2}-M^{2}}$ the nucleon energy and momentum, $E_{\max }=E_{\Lambda}-m_{\pi}$, the maximum nucleon energy, neglecting the recoil of the nucleus, and $R_{l j}(p ; r)$ a continuum solution of the nucleon Schrödinger equation [32], with the same potential as that used for the bound nucleons to compute the discrete contribution to the decay width. The nucleon wave function normalization in the continuum is the same as that given above for the pions (Eqs. (22-23)).

As a test of the multipole expansion in the continuum, we recovered the free space $\Lambda_{-}^{-}$ decay width of Eq. (8) from the multipolar expansion of Eqs. (14) to (26). For this purpose 
we set $A=1$ and replaced the radial pion and nucleon wave functions by spherical Bessel's functions, $j_{l}$, and the radial $\Lambda$-wave function by $\sqrt{4 \pi / V}$, being $V=4 \pi L^{3} / 3$ the volume of interaction, which will be finally sent to infinity when calculating physical observables. The test is straightforward, taking into account:

$$
\begin{aligned}
\sum_{l=0}^{\infty}(2 l+1) & \left|\sqrt{\frac{4 \pi}{V}} \int_{0}^{L} d r r^{2} j_{l}(p r) j_{l}(q r)\right|^{2} \\
& =\frac{4 \pi}{V} \int_{0}^{L} d r r^{2}\left(\sum_{l=0}^{\infty}(2 l+1) j_{l}^{2}(p r)\right) \int_{0}^{L} d r r^{2} j_{l}(p r) j_{l}(q r)+\mathcal{O}(1 / L) \\
& =\frac{\pi}{2 p^{2}} \delta(p-q)+\mathcal{O}(1 / L)
\end{aligned}
$$

On the other hand, we have also used this free space limit to test our computational code and the precision of our numerical algorithms to solve differential equations, to perform integrations and the sum over an infinite number of multipoles. The idea was, taking as starting point the formulae of Eqs. (14) to (26) with a radial $\Lambda$ - wave function given by

$\sqrt{4 \pi / V}$ and switching off the pion-nucleus, and the nucleon potentials, to recover numerically, in the limit $L$ going to infinity, the free space $\Lambda$ decay width. Such a calculation, from the numerical point of view, is much more demanding that the actual calculation of the pionic decay width of the hypernuclei, since the Dirac's delta appearing in Eq. (27) has to be constructed numerically out of a large sum over multipoles and slowly convergent integrals.

\section{$3 \quad$ Nuclear Structure and Energy Balance}

We model the nuclear structure of the ${ }^{A-1} Z$ system by a Slater determinant built with single-particle wave functions obtained by diagonalizing a WS potential well

$$
V_{\mathrm{WS}}(r)=V_{0} f\left(r, R_{0}, a_{0}\right)+V_{L S} \frac{\vec{l} \cdot \vec{\sigma}}{r} \frac{d f\left(r, R_{L S}, a_{L S}\right)}{d r}+\tilde{V}_{C}(r),
$$

where

$$
f(r, R, a)=\frac{1}{1+\exp \left(\frac{r-R}{a}\right)}
$$

and $\tilde{V}_{C}(r)$ is the Coulomb potential created by an homogeneous charge distribution of radius $R_{C}$. The parameters, compiled in Table 2 for neutrons and Table 3 for protons, of this potential are adjusted ${ }^{3}$ to reproduce some experimental single-particle energies around the Fermi level [34]-[35]. This will enforce not only a correct energy balance for the decay process to the first available shell but also to some excited states. The main contributions to the processes of Eqs. (1) and (2) come from situations where the

\footnotetext{
${ }^{3}$ Essentially, we adjust the depths $V_{0}$ and $V_{L S}$, and for the radius- and thickness-type parameters standard values have been used
} 


\begin{tabular}{ccccccc}
\hline & $V_{0}[\mathrm{MeV}]$ & $R_{0}[\mathrm{fm}]$ & $a_{0}[\mathrm{fm}]$ & $V_{L S}[\mathrm{MeV}]$ & $R_{L S}[\mathrm{fm}]$ & $a_{L S}[\mathrm{fm}]$ \\
\hline${ }^{11} \mathrm{C}$ & -57.0 & 2.86 & 0.53 & -6.05 & 2.86 & 0.53 \\
${ }^{27} \mathrm{Si}$ & -66.2 & 3.50 & 0.70 & -3.30 & 3.75 & 0.70 \\
${ }^{55} \mathrm{Fe}$ & -54.0 & 4.70 & 0.50 & -8.30 & 4.70 & 0.50 \\
\hline
\end{tabular}

Table 2: Neutron WS parameters used in this work.

\begin{tabular}{cccccccc}
\hline & $V_{0}[\mathrm{MeV}]$ & $R_{0}[\mathrm{fm}]$ & $a_{0}[\mathrm{fm}]$ & $V_{L S}[\mathrm{MeV}]$ & $R_{L S}[\mathrm{fm}]$ & $a_{L S}[\mathrm{fm}]$ & $R_{C}[\mathrm{fm}]$ \\
\hline${ }^{11} \mathrm{C}$ & -38.4 & 2.86 & 0.53 & -6.05 & 2.86 & 0.53 & 2.86 \\
${ }^{27} \mathrm{Si}$ & -47.4 & 3.75 & 0.53 & -10.0 & 3.75 & 0.53 & 3.75 \\
${ }^{55} \mathrm{Fe}$ & -50.8 & 4.70 & 0.50 & -8.30 & 4.70 & 0.50 & 4.70 \\
\hline
\end{tabular}

Table 3: Proton WS parameters used in this work.

daughter nucleus is left in the ground state or in the first few excited states. Since the effect of the Pauli blocking depends strongly on the pion energy after the decay, it is important, as shown in Ref. [16], to perform a correct balance of energies, using when possible experimental energies.

Thus, the energy of the first non-occupied shell is fixed to the mass difference between the ground states of the ${ }^{A} Z$ and ${ }^{A-1} Z$ nuclei for the case of neutron $\left(\pi^{0}\right)$ decay and of the ${ }^{A}(Z+1)$ and ${ }^{A-1} Z$ for the case of the proton $\left(\pi^{-}\right)$decay channel. In Table 4 we give the first available shells and their empirical energies obtained in this way. The energies of this table do not totally determine the parameters of the mean field nucleon potential, and one has still the possibility to fit some excited state energies. In what follows, we give some more details of the adjusted shells.

\begin{tabular}{c|cc|cc}
\hline Hypernucleus & Neutron Shell & Energy $[\mathrm{MeV}]$ & Proton Shell & Energy $[\mathrm{MeV}]$ \\
\hline${ }_{\Lambda}^{12} \mathrm{C}$ & $1 p_{3 / 2}$ & -18.72 & $1 p_{1 / 2}$ & -0.60 \\
${ }_{\Lambda}^{28} \mathrm{Si}$ & $1 d_{5 / 2}$ & -17.18 & $2 s_{1 / 2}$ & -2.07 \\
${ }_{\Lambda}^{56} \mathrm{Fe}$ & $2 p_{3 / 2}$ & -11.20 & $1 f_{7 / 2}$ & -5.85 \\
\hline
\end{tabular}

Table 4: Single particle energies for the first available, to the pionic decay of the hypernuclei studied in this work, nucleon shells and their empirical binding energies. Energies have been obtained from the neutron and proton separation energies of the nuclear ${ }^{A} Z$ and ${ }^{A}(Z+1)$ spices respectively, and have been taken from Ref. [36]. 
- ${ }_{\Lambda}^{12} \mathrm{C}$ : We assume a closed proton and neutron $1 p_{3 / 2}$ shell configurations for the ground state of ${ }^{12} \mathrm{C}$ and a $1 p_{1 / 2}$ (proton) $1 p_{3 / 2}^{-1}$ (neutron) configuration for the ground state of ${ }^{12} \mathrm{~N}$. The rest of neutron and proton shells adjusted by the mean field potentials, which parameters are given in Tables 2 and 3, are:

Neutrons: We fix the energy of the $1 p_{1 / 2}$ neutron shell from the energy of the first excited state of ${ }^{12} \mathrm{C}\left[2^{+}{ }^{12} \mathrm{C}^{*}(4.43) \mathrm{MeV}\right]$. We assume a particle-hole configuration $1 p_{1 / 2}, 1 p_{3 / 2}^{-1}$ which leads to a binding energy for the $1 p_{1 / 2}$ shell of $-14.29 \mathrm{MeV}$. We are aware that this excited state of ${ }^{12} \mathrm{C}$ might also have some contributions from proton degrees of freedom, configuration mixing, $2 p 2 h$ or higher excitations, collective degrees of freedom, etc, not considered in this simple model. However, as long as this state had a sizeable component $\left|1 p_{1 / 2}, 1 p_{3 / 2}^{-1}\right\rangle$, it would be reachable in the weak decay of the hypernucleus and the procedure described above would guarantee that the energy balance is correct, not only when the ${ }^{12} \mathrm{C}$ is left on its ground state, but also when it is left on its first excited state, leading then in both cases to good pion wave-functions. The nuclear matrix elements appearing in the evaluation of the decay width, though depending strongly on the pion wave-function, are less sensitive to the specific details of the nuclear wave function.

Protons: There are no ${ }^{12} \mathrm{~N}$ excited states amenable to be explained within a shell model, since all levels compiled in Ref. [36] are broad resonant states populated in nuclear reactions. Therefore, we choose to use the same spin-orbit force as in the neutron case, and fix the depth of the central part of the potential to reproduce the $1 p_{1 / 2}$ shell as given in Table 4 . The mean field potential adjusted in this way, does not provide excited states for ${ }^{12} \mathrm{~N}$.

- ${ }_{\Lambda}^{28} \mathrm{Si}$ : We assume closed proton and neutron $1 d_{5 / 2}$ shell configurations for the ground state of ${ }^{28} \mathrm{Si}$ and a $2 s_{1 / 2}$ (proton) $1 d_{5 / 2}^{-1}$ (neutron) configuration for the ground state of ${ }^{28} \mathrm{P}$. The rest of neutron and proton shells adjusted by the mean field potentials are:

Neutrons: We fix the energy of the $2 s_{1 / 2}$ neutron shell from the energy of the first excited state of ${ }^{28} \mathrm{Si}\left[2^{+}{ }^{28} \mathrm{Si}^{*}(1.78) \mathrm{MeV}\right]$. We assume a neutron particle-hole configuration of the type $\left|2 s_{1 / 2}, 1 d_{5 / 2}^{-1}\right\rangle$ which leads to a binding energy for the $2 s_{1 / 2}$ shell of $15.40 \mathrm{MeV}$. Limitations and virtues of describing the mesonic decay with this simple picture for the underlying nuclear core, are similar to those commented above for the ${ }_{\Lambda}^{12} \mathrm{C}$ case. We should mention that to adjust the WS neutron potential to simultaneously give the empirical $1 d_{5 / 2}$ and $2 s_{1 / 2}$ energy shells, keeping the $1 d_{3 / 2}$ energy shell above the $2 s_{1 / 2}$, is delicate and that we had to use a value a bit high $(0.70 \mathrm{fm})$ for the thickness parameters $a_{0}$ and $a_{L S}$. We have also tried smaller values for the thickness. For instance for values of $a_{0}=a_{L S}=0.58 \mathrm{fm}$, in order to adjust the empirical energies of both the $1 d_{5 / 2}$ and $2 s_{1 / 2}$ shells, a spin orbit force very small $\left(V_{L S}=-0.1 \mathrm{MeV}\right.$ with $\left.V_{0}=-65.75 \mathrm{MeV}\right)$ is required. Despite that both the $1 d_{5 / 2}$ and $1 d_{3 / 2}$ shells turn out to be almost degenerate, and that the latter one is now 
deeper than the $2 s_{1 / 2}$ shell, the decay width is rather stable, and it gets increased only by about $10 \%$, with respect to the results of the next section. This increase is due to an enhancement of the $1 d_{3 / 2}$ shell contribution, but the corresponding configuration $\left|1 d_{3 / 2}, 1 d_{5 / 2}^{-1}\right\rangle$ has an excitation energy too small to correspond to any experimental excited state of ${ }^{28} \mathrm{Si}$.

Protons: For the ${ }^{28} \mathrm{P}$ nucleus, as for the case of ${ }^{12} \mathrm{~N}$, there are no excited states amenable to be explained within a shell model, since all levels compiled in Ref. [36] are again broad resonant states populated in nuclear reactions. We fix the depth of the central part of the potential to reproduce the $2 s_{1 / 2}$ shell as given in Table 4 and we choose the depth of the spin-orbit such that the next shell $\left(1 d_{3 / 2}\right)$ appears in the continuum. We will discuss in the next section, the dependence of our results on the precise value of the spin-orbit force. If we use instead the same spin-orbit force as in the neutron case, there will appear excited ${ }^{28} \mathrm{P}$ states, which cannot be identified in the experiment.

- ${ }_{\Lambda}^{56} \mathrm{Fe}$ : The nuclear core structure of this hypernucleus is more difficult to describe, within our simple shell model, than those of ${ }_{\Lambda}^{28} \mathrm{Si}$ and ${ }_{\Lambda}^{12} \mathrm{C}$ hypernuclei. Thus, our results for the decay of this hypernucleus are subject to more theoretical uncertainties. In what the ground states respect, for ${ }^{56} \mathrm{Fe}$, we assume a configuration composed of two paired $1 f_{7 / 2}$ proton holes and two paired $2 p_{3 / 2}$ neutron particles, while for ${ }^{56} \mathrm{Co}$, we assume a proton hole in the $1 f_{7 / 2}$ shell and a neutron in the $2 p_{3 / 2}$ shell.

The rest of neutron and proton shells adjusted by the mean field potentials are:

Neutrons: We fix the energy of the $1 f_{5 / 2}$ neutron shell from the energy of the first excited state of ${ }^{56} \mathrm{Fe}\left[2^{+56} \mathrm{Fe}^{*}(0.847) \mathrm{MeV}\right]$. We assume for neutrons a two particle configuration $\left|2 p_{3 / 2}, 1 f_{5 / 2}\right\rangle$ which leads to a binding energy for the $1 f_{5 / 2}$ shell of $10.35 \mathrm{MeV}$.

Protons: The first excited state of ${ }^{56}$ Co has spin-parity $3^{+}$and an excitation energy of $0.16 \mathrm{MeV}$. In principle, one might use it to determine properties of the WS proton mean field potential. However, a word of caution must be said here. One might try to describe this state as a proton configuration with two paired holes in the $1 f_{7 / 2}$ shell and a particle in the $2 p_{3 / 2}$ shell, in such a way that the above configuration would determine the energy of the $2 p_{3 / 2}$ shell. The $1 f_{7 / 2}$ shell completes 28 protons which is a magic number, and thus one expects an energy gap between this shell and the following in energy, $2 p_{3 / 2}$, appreciable and of the order of the $\mathrm{MeV}$, and not as small as $0.16 \mathrm{MeV}$. Then, likely, the $3^{+}$excited state should have an important neutron component (one neutron in the $2 p_{3 / 2}$ shell and the other in the $1 f_{5 / 2}$ one) or more complex components not considered in our simple shell model. Then, it seems safe to guarantee that this state will not have a sizeable component $\left|2 p_{3 / 2}, 1 f_{7 / 2}^{-2}\right\rangle$. Therefore, we choose to use the same spin-orbit force as in the neutron case and fix the depth of the central part of the potential to reproduce the $1 f_{7 / 2}$ shell as given 


\begin{tabular}{|c|c|c|c|c|c|c|c|c|c|}
\hline & & \multicolumn{4}{|c|}{$\pi^{0}$ Decay } & \multicolumn{4}{|c|}{$\pi^{-}$Decay } \\
\hline${ }_{\Lambda}^{A} \mathrm{Z}$ & $V_{\mathrm{opt}}$ & $\Gamma_{d}$ & $\Gamma_{c}$ & $\Gamma$ & $\Gamma_{\exp }[3]$ & $\Gamma_{d}$ & $\Gamma_{c}$ & $\Gamma$ & $\Gamma_{\exp }[7]$ \\
\hline \multirow{2}{*}{${ }_{\Lambda}^{12} \mathrm{C}$} & $\mathrm{FP}$ & 0.136 & 0.008 & 0.144 & \multirow{2}{*}{$0.217 \pm 0.084$} & 0.079 & 0.027 & 0.106 & \multirow{2}{*}{$0.113 \pm 0.013 \pm 0.005$} \\
\hline & NQ & 0.150 & 0.008 & 0.158 & & 0.082 & 0.028 & 0.110 & \\
\hline \multirow{2}{*}{${ }_{\Lambda}^{28} \mathrm{Si}$} & FP & 0.061 & 0.001 & 0.062 & & 0.018 & 0.019 & 0.037 & \multirow{2}{*}{$0.047 \pm 0.008 \pm 0.002$} \\
\hline & NQ & 0.074 & 0.001 & 0.075 & & 0.020 & 0.019 & 0.039 & \\
\hline \multirow{2}{*}{${ }_{\Lambda}^{56} \mathrm{Fe}$} & FP & 0.010 & 0.003 & 0.013 & & 0.005 & 0.009 & 0.014 & \multirow{2}{*}{$<0.015$ (90\%C.L.) } \\
\hline & NQ & 0.010 & 0.003 & 0.013 & & 0.004 & 0.010 & 0.014 & \\
\hline
\end{tabular}

Table 5: Pionic decay widths, units of $\Gamma_{\Lambda}$, calculated with two different pion nucleus optical potentials: FP stands for the full optical potential of Ref. [25], NQ stands for the pion-nucleus interaction obtained by switching off the imaginary part of the FP optical potential coming from quasielastic pion scattering.

in Table 4. The mean field potential adjusted in this way, leads to an excited state of about $5 \mathrm{MeV}$ above the ground state.

\section{Results and Concluding Remarks}

Results for the pionic decay widths of ${ }_{\Lambda}^{12} \mathrm{C},{ }_{\Lambda}^{28} \mathrm{Si}$ and ${ }_{\Lambda}^{56} \mathrm{Fe}$, calculated with two different pion nucleus optical potentials are presented in Table 5. The effect of the imaginary part of the potential is to remove from the emerging pion flux those pions which undergo quasielastic scattering or pion absorption. However, while the pions absorbed should be definitely removed, this is not the case with those which undergo quasielastic scattering, since even if they collide, they are still there and will be observed. This means that one should not remove these pions from the pion flux and we take this into account here. The effect is moderately small, as it was already noted in Ref. [16].

The agreement with the recent KEK measurements is remarkably good and it is also good when our results are compared to the older measurement of the $\pi^{0}$ decay width of ${ }_{\Lambda}^{12} \mathrm{C}$. This is a clear success of the model of Refs. [24, 25] to account for the pionnucleus dynamics at low energies. In Ref. [16], it was obtained a value of $0.086 \Gamma_{\Lambda}$ for the $\pi^{-}{ }_{-}^{12} \mathrm{C}$ decay width. In this work we find a value about $25 \%$ higher and in a closer agreement with the experiment. Differences are even bigger if one looks at the continuum contribution and also appear for the $\pi^{0}$ decay case. In both works, here and in that of Ref. [16], the same $\pi^{-}$-wave function has been used, being then the difference due to an improved treatment of the underlying nuclear core dynamics. As we will see below, the barrier method employed in Ref. [16] to estimate the continuum contribution compare reasonably well to the more correct treatment followed here, when the same nuclear potential is used. The discrepancies have to be attributed not only to the different WS potentials used in both works, but also to the somewhat artificial procedure followed in Ref. [16] to enforce the correct energy balance in the decay. 

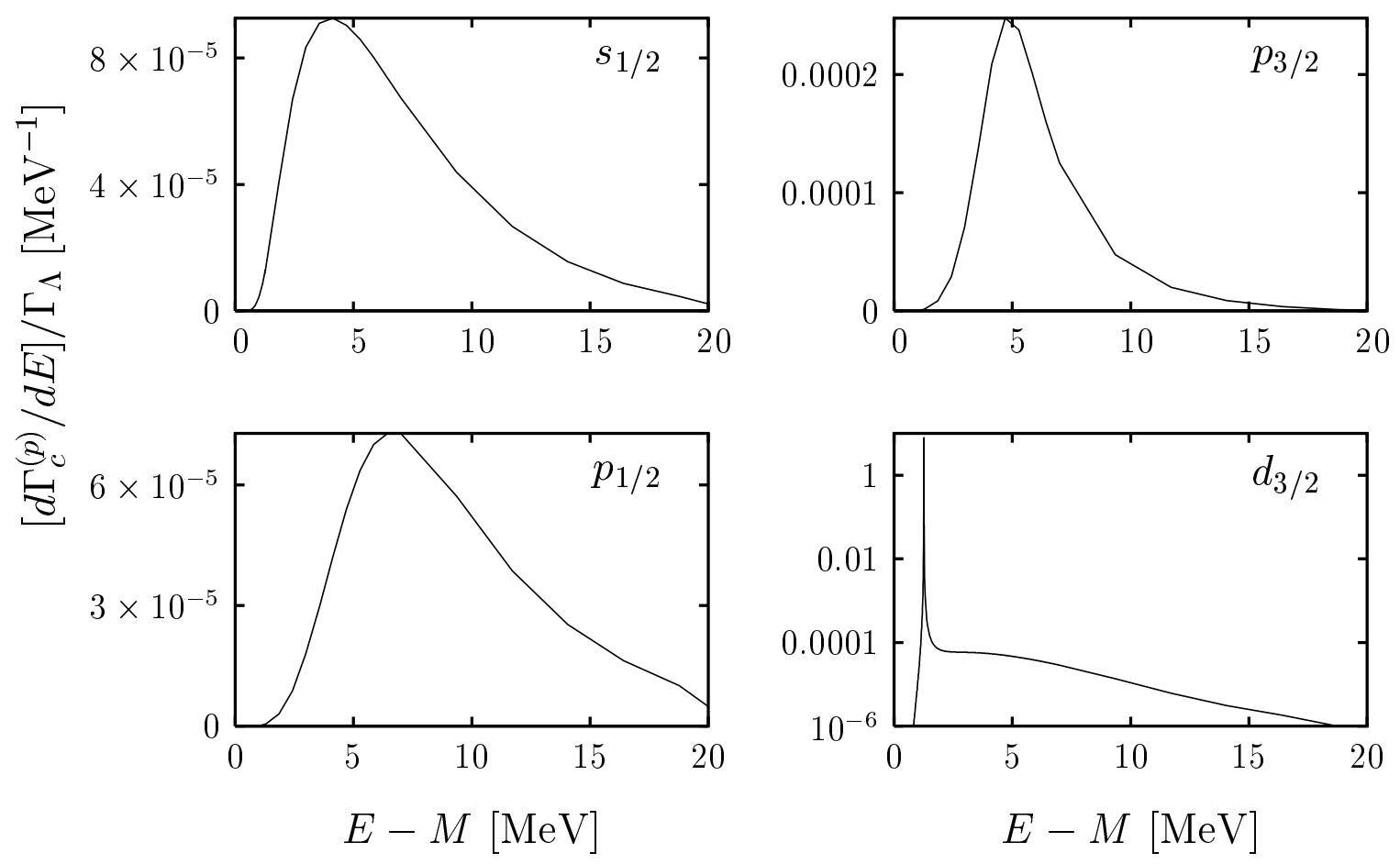

Figure 1: Continuum proton energy distributions from the $\pi^{-}$- decay width of ${ }_{\Lambda}^{28} \mathrm{Si}$. Results have been obtained with the NQ $\pi^{-}$-nucleus optical potential, as defined in the caption of Table 5 , and only the most relevant multipoles are shown.

Note that in Table 5 the computed widths are always slightly below the data. The contribution of other reaction channels, not included in Eqs. (1-4), though we expect it to be small, would improve the present calculation.

In contrast to most of the hypernuclei studied previously by using the WFM [13]-[17], for the three hypernuclei considered here, the continuum contribution in some cases plays a crucial role. As a matter of example, for $\pi^{-}$decay of ${ }_{\Lambda}^{28} \mathrm{Si}$, it turns out that $\Gamma_{c}$ is of the same size as $\Gamma_{d}(0.020$ vs 0.019$)$ and essential to understand the experimental datum. This reinforces the need of updating the calculation of Ref. [16], where the evaluation of the continuum contribution was a bit rough, since there was assumed that it was only a small fraction of the total. Indeed, in Ref. [16], the continuum contribution was estimated by discretizing the positive nucleon energy levels, by means of an infinite barrier placed at distances of about $20 \mathrm{fm}$. In Table 6 we compare the CSM of this work with the model of Ref. [16]. In both cases we use the same nucleon WS mean potential. Both methods agree remarkably well and much better of what one might expect a priory. Within the model of Ref. [16], the sizeable contribution of the continuum is due to the presence of a quasi-bound $(1.27 \mathrm{MeV})$ state, $1 d_{3 / 2}$ and it has its counterpart in the size of the $d_{3 / 2}$ multipole in the CSM. Even more, the differential partial width $d \Gamma_{d_{3 / 2}}^{(p)} / d E$ presents a narrow peak (resonance), which gives most of the contribution to the integrated partial width, located around $1.27 \mathrm{MeV}$, as can be seen in Fig 1. Small changes in the WS proton mean potential can bind this shell, going then its important contribution to the 

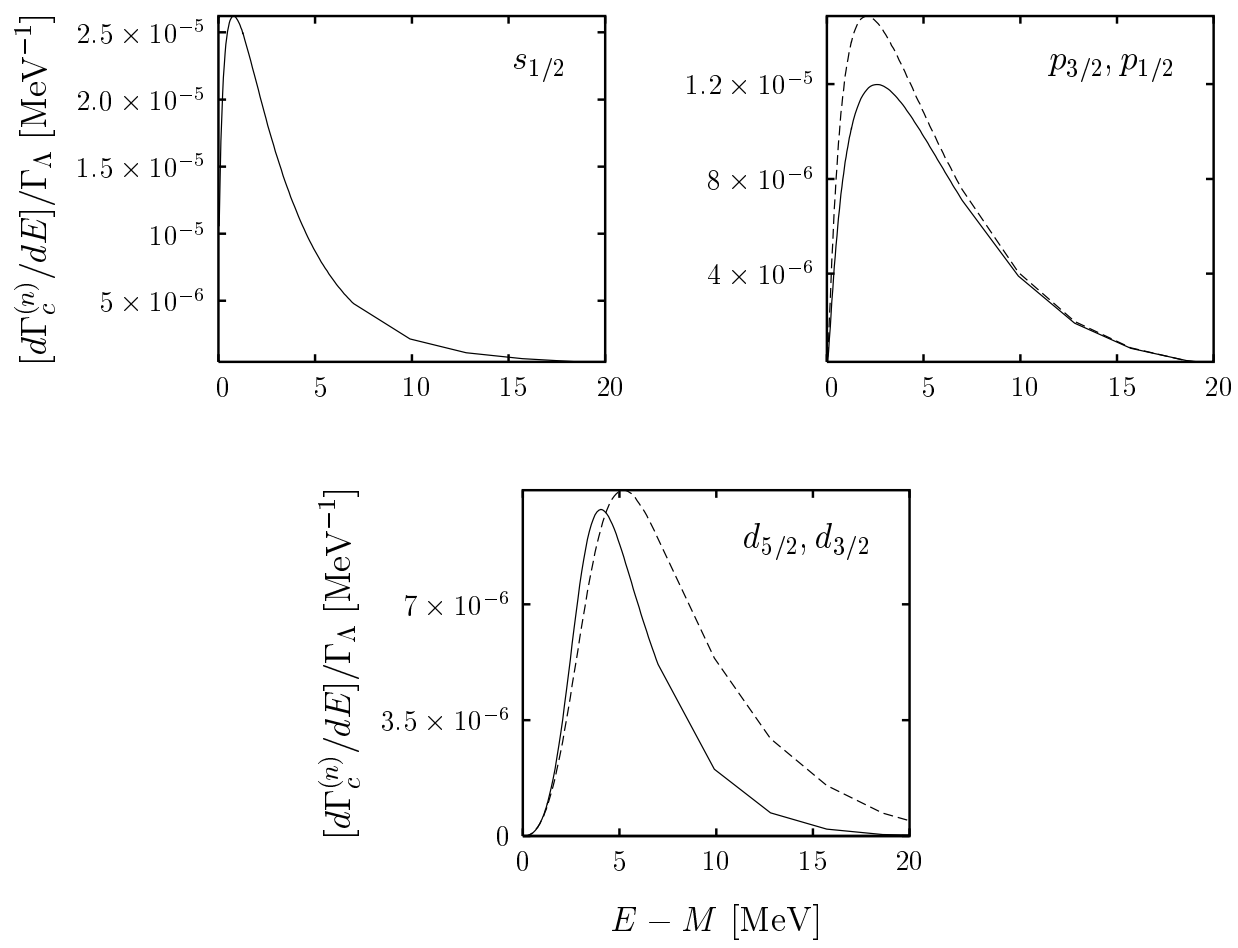

Figure 2: Neutron energy distributions for ${ }_{\Lambda}^{28} \mathrm{Si}$-decay. Solid (dashed) line corresponds to the $j=l+1 / 2$ $(j=l-1 / 2)$ multipole. The integrated decay widths are $1.2 \times 10^{-4}, 1.1 \times 10^{-4}, 1.0 \times 10^{-4}, 9 \times 10^{-5}$, and $6 \times 10^{-5}$, in units of $\Gamma_{\Lambda}$, for the $1 s_{1 / 2}, 1 p_{1 / 2}, 1 p_{3 / 2}, 1 d_{3 / 2}$ and $1 d_{5 / 2}$ multipoles respectively.

discrete part, $\Gamma_{d}$, but remaining the total width $\Gamma_{d}+\Gamma_{c}$ almost unchanged. For example, if one uses a spin-orbit force depth of $-7 \mathrm{MeV}$ instead of $-10 \mathrm{MeV}$, the $1 d_{3 / 2}$ proton shell becomes bound $(-0.1 \mathrm{MeV})$ and the total width is $0.042 \Gamma_{\Lambda}$ instead of the value of $0.039 \Gamma_{\Lambda}$ quoted in Table 5 . In the model of Ref. [16], the exact position of the barrier might influence energies, the number of shells, and contributions of each shell, but again the total contribution to $\Gamma_{c}$ remains rather constant, as long as the barrier is placed far enough.

We have tested for the sensitivity of our results to the mean field nucleon WS parameters. Thus, we have increased and decreased the spin-orbit depth $V_{L S}$ by $10 \%$ and re-adjusted the depth of the central part of the nucleon potential, $V_{0}$, to get the experimental ground states masses of the involved nuclei, it is to say $V_{0}$ is modified to reproduce again the energies given in Table 4, with the new values of the spin-orbit force. Results turn out to be quite stable, changing at most by a $2 \%$, except for the $\pi^{-}$- decay of ${ }_{\Lambda}^{56} \mathrm{Fe}$, where the uncertainty can be as large as one part in fifteen.

To finish, we show the continuum nucleon energy distributions for the first nucleon multipoles, which give the bulk of the total, (Figs. 1 to 4) and the contribution to $\Gamma_{d}$ of each of the unoccupied shells (Table 7) for the $\pi^{-}$and $\pi^{0}$ decay widths of ${ }_{\Lambda}^{12} \mathrm{C}$, of ${ }_{\Lambda}^{28} \mathrm{Si}$ and of ${ }_{\Lambda}^{56} \mathrm{Fe}$. All results have been obtained using the NQ $\pi$-nucleus optical potential. Resonances appear as distinctive features in the continuum contribution of some multipoles. 

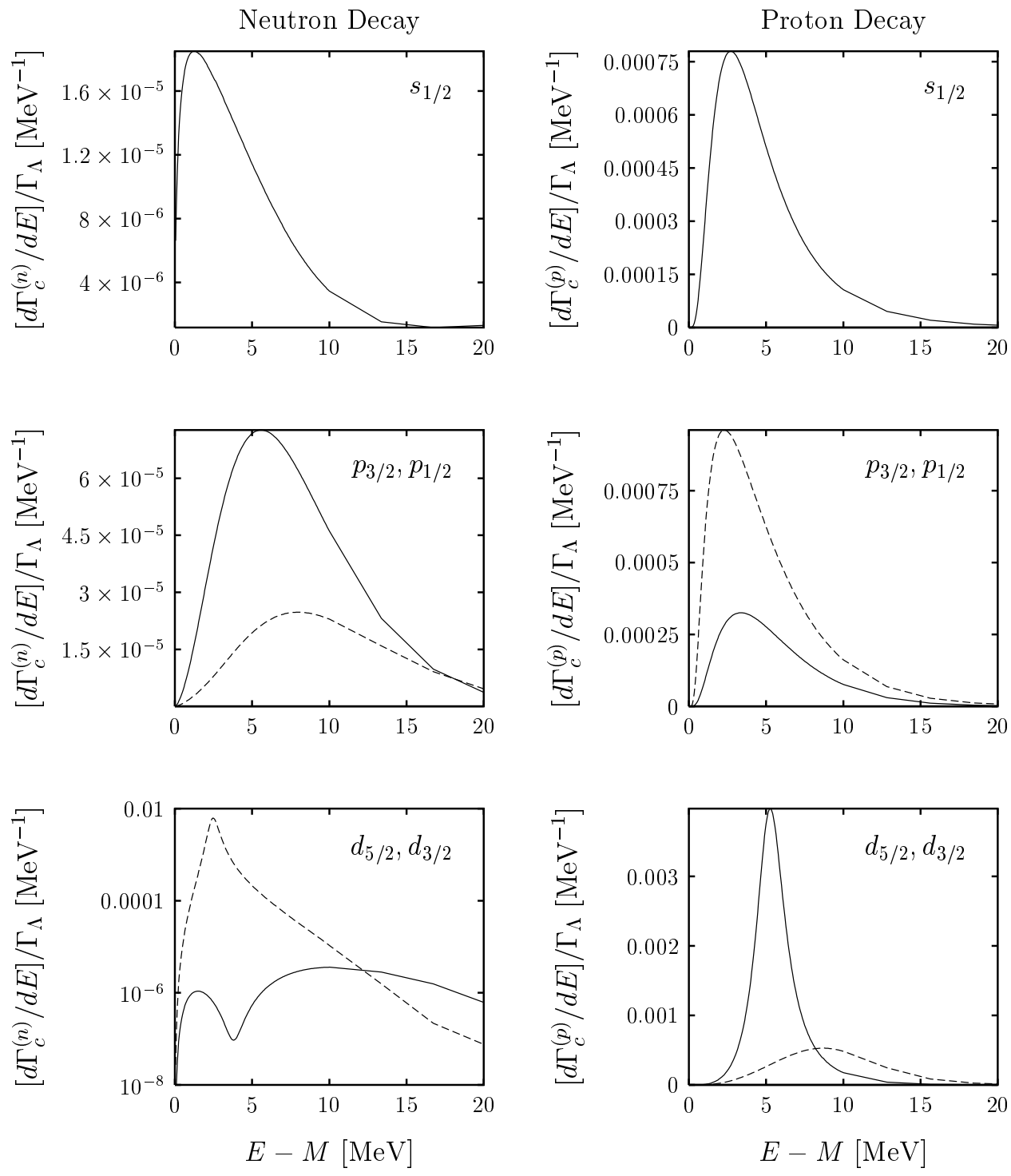

Figure 3: Neutron (left) and proton (right) energy distribution for ${ }_{\Lambda}^{12} \mathrm{C}$-decay. Solid (dashed) line corresponds to the $j=l+1 / 2(j=l-1 / 2)$ multipole. The neutron integrated decay widths are $1.4 \times 10^{-4}, 3.0 \times 10^{-4}, 7.1 \times 10^{-4}, 6.62 \times 10^{-3}$, and $4 \times 10^{-5}$, in units of $\Gamma_{\Lambda}$, for the $1 s_{1 / 2}, 1 p_{1 / 2}$, $1 p_{3 / 2}, 1 d_{3 / 2}$ and $1 d_{5 / 2}$ multipoles respectively. While for protons, the contribution of the multipoles are $4.33 \times 10^{-3}, 5.79 \times 10^{-3}, 2.16 \times 10^{-3}, 4.30 \times 10^{-3}$, and $1.124 \times 10^{-2}$. 

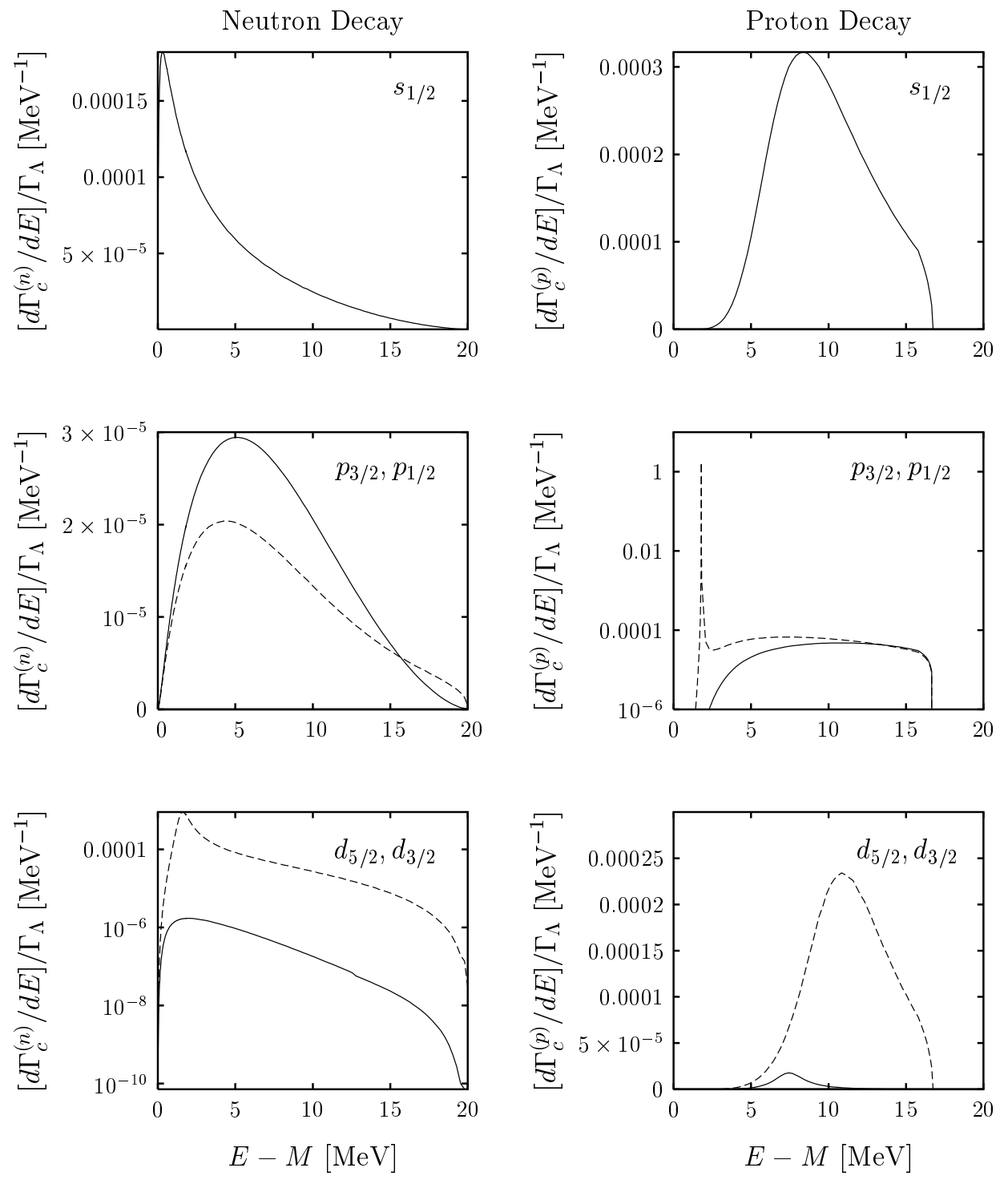

Figure 4: The same as Fig. 3 for ${ }_{1}^{56}$ Fe-decay. The neutron integrated decay widths are $8.2 \times 10^{-4}$, $2.3 \times 10^{-4}, 3.2 \times 10^{-4}, 1.56 \times 10^{-3}$, and $1 \times 10^{-5}$, in units of $\Gamma_{\Lambda}$, for the $1 s_{1 / 2}, 1 p_{1 / 2}, 1 p_{3 / 2}, 1 d_{3 / 2}$ and $1 d_{5 / 2}$ multipoles respectively. While for protons, the contribution of the multipoles are $2.47 \times 10^{-3}$, $5.21 \times 10^{-3}, 4.8 \times 10^{-4}, 1.49 \times 10^{-3}$, and $5 \times 10^{-5}$. 


\begin{tabular}{|c|c|c|c|c|}
\hline \multicolumn{2}{|c|}{ This work } & \multicolumn{3}{|c|}{ Barrier at $20 \mathrm{fm}[16]$} \\
\hline Multipole & {$\left[\Gamma_{l j}^{(p)}\right]$} & Shell & Energy $[\mathrm{MeV}]$ & {$\left[\Gamma_{n l j}^{(p)}\right]$} \\
\hline \multirow[b]{4}{*}{$s_{1 / 2}$} & & $3 s_{1 / 2}$ & 2.45 & 0.0002 \\
\hline & & $4 s_{1 / 2}$ & 5.19 & 0.0003 \\
\hline & & $5 s_{1 / 2}$ & 9.21 & 0.0002 \\
\hline & 0.0007 & Total $s_{1 / 2}$ & & 0.0007 \\
\hline \multirow[b]{3}{*}{$p_{3 / 2}$} & & $3 p_{3 / 2}$ & 4.59 & 0.0005 \\
\hline & & $4 p_{3 / 2}$ & 7.25 & 0.0004 \\
\hline & 0.0011 & Total $p_{3 / 2}$ & & 0.0009 \\
\hline \multirow[b]{3}{*}{$p_{1 / 2}$} & & $3 p_{1 / 2}$ & 5.02 & 0.0002 \\
\hline & & $4 p_{1 / 2}$ & 8.14 & 0.0002 \\
\hline & 0.0007 & Total $p_{1 / 2}$ & & 0.0004 \\
\hline \multirow[b]{4}{*}{$d_{3 / 2}$} & & $1 d_{3 / 2}$ & 1.27 & 0.0160 \\
\hline & & $2 d_{3 / 2}$ & 3.22 & 0.0001 \\
\hline & & $3 d_{3 / 2}$ & 6.31 & 0.0001 \\
\hline & 0.0165 & Total $p_{1 / 2}$ & & 0.0162 \\
\hline \multicolumn{2}{|c|}{$\Gamma_{c}=0.019$} & \multicolumn{3}{|c|}{$\Gamma_{c}=0.018$} \\
\hline
\end{tabular}

Table 6: Continuum contributions to the pionic decay width, units of $\Gamma_{\Lambda}$, calculated with the NQ $\pi^{-}$-nucleus optical potential, as defined in the caption of Table 5, and two different methods: the sum of multipoles of the type $\Gamma_{l j}^{(p)}$ defined in Eqs. (14) and (25) and the sum over positive energy discrete bound (by the effect of an infinite barrier placed at $20 \mathrm{fm}$ ) states [16]. Only contributions to $\Gamma_{c}$ larger than $5 \times 10^{-4} \Gamma_{\Lambda}$ are shown. Results are for the $\pi^{-}$-decay of the ${ }_{\Lambda}^{28} \mathrm{Si}$ hypernucleus. 


\begin{tabular}{c|ccc|ccc}
\hline & \multicolumn{3}{|c|}{$\pi^{0}$ Decay } & \multicolumn{3}{c}{$\pi^{-}$Decay } \\
\hline${ }_{\Lambda}^{A} \mathrm{Z}$ & Shell & Energy $[\mathrm{MeV}]$ & {$\left[\Gamma_{n l j}^{(n)}\right]_{d}$} & Shell & Energy $[\mathrm{MeV}]$ & {$\left[\Gamma_{n l j}^{(p)}\right]_{d}$} \\
\hline & $1 p_{3 / 2}$ & -18.72 & 0.0473 & & & \\
& $1 p_{1 / 2}$ & -14.29 & 0.0865 & $1 p_{1 / 2}$ & -0.60 & 0.0817 \\
& $1 d_{5 / 2}$ & -3.51 & 0.0156 & & & \\
& $2 s_{1 / 2}$ & -2.07 & 0.0008 & & Total & 0.082 \\
\hline${ }_{\Lambda}^{12} \mathrm{C}$ & & Total & 0.150 & & & \\
\hline & $1 d_{5 / 2}$ & -17.18 & 0.0086 & & & \\
& $2 s_{1 / 2}$ & -15.40 & 0.0285 & $2 s_{1 / 2}$ & -2.07 & 0.0204 \\
& $1 d_{3 / 2}$ & -14.76 & 0.0342 & & & \\
& $2 p_{3 / 2}$ & -3.29 & 0.0009 & & & 0.020 \\
& $1 f_{7 / 2}$ & -2.95 & 0.0010 & & & 0.0013 \\
& $2 p_{1 / 2}$ & -2.35 & 0.0009 & & Total & \\
${ }_{\Lambda}^{28} \mathrm{Si}$ & \multicolumn{7}{|c|}{ Total } & 0.074 & & -0031 \\
& & -11.20 & 0.0029 & $2 p_{3 / 2}$ & -0.37 & \\
& $2 p_{3 / 2}$ & -10.35 & 0.0027 & & & 0.004 \\
\hline & $1 f_{5 / 2}$ & -8.84 & 0.0035 & & &
\end{tabular}

Table 7: Discrete contributions to the pionic decay width (units of $\Gamma_{\Lambda}$ ) for each of the unoccupied shells, calculated with the NQ $\pi$-nucleus optical potential, as defined in the caption of Table 5 . 
The study of the mesonic decay of $\Lambda \Lambda$ hypernuclei constitutes an obvious extension of this work. To improve the existing calculations [33, 37] would require a correct treatment of the $\Lambda \Lambda$ pair inside of the nuclear medium. Thus, the recent work of Ref. [38], where short and long range correlations are taken into account, will be a good starting point for this end.

\section{Acknowledgments}

This research was supported by DGI and FEDER funds, under contract BFM2002-03218 and by the Junta de Andalucía (Spain). C. Albertus wishes to acknowledge a PhD fellowship from Junta de Andalucía.

\section{References}

[1] A. Montwill, et al., Nucl. Phys. A234 (1974) 413.

[2] R.Grace, et al., Phys. Rev. Lett. 55 (1985) 1055.

[3] A.Sakaguchi, et al., Nuovo Cimento 102A (1989) 511.

[4] P.D. Barnes, Nucl. Phys. A450 (1986) 43c; A478 (1988) 127c.

[5] J.J. Szymanski et al., Phys. Rev. C43 (1991) 849.

[6] A. Sakaguchi et al., Phys. Rev. C43 (1991) 73.

[7] Y. Sato, et al., Nucl. Phys. A691 (2001) 189c.

[8] R.H. Dalitz, Phys. Rev. 112 (1958) 605.

[9] R.H. Dalitz and L.Liu, Phys. Rev. 116 (1959) 1312.

[10] Y.Kurihara, Y.Akaishi and H.Tanaka, Phys. Rev. C31 (1985) 971.

[11] H.Bando and H.Takaki, Progr. Theor. Phys. 72 (1984) 106; H. Bando, T. Motoba and J. Zofka, Int. Jour. Mod. Phys. A5 (1990) 4021.

[12] E.Oset and L.L.Salcedo, Nucl. Phys. A443 (1985) 704.

[13] K.Itonaga, T.Motoba and H.Bando, Zeits. Phys. A330 (1988) 209.

[14] T.Motoba, K.Itonaga and H.Bando, Nucl. Phys. A489 (1988) 683.

[15] T. Motoba, Nucl. Phys. A527 (1991) 485c; ibid, Few Body Systems, Suppl. 5 (1992) 386; ibid, Nucl. Phys. A547 (1992) 115c.

[16] J. Nieves and E. Oset, Phys. Rev. C47 (1993) 1478. 
[17] U. Straub, J. Nieves, A. Faessler and E.Oset, Nucl. Phys. A556 (1993) 531.

[18] A. Ramos, E. Oset and L.L. Salcedo, Phys. Rev. C50 (1994) 2314.

[19] E. Oset, P. Fernández de Córdoba, J. Nieves, A. Ramos and L.L. Salcedo, Prog. Theor. Phys. Suppl. 117 (1994) 461.

[20] T. Motoba and K. Itonaga, Prog. Theor. Phys. Suppl. 117 (1994) 477.

[21] L. Zhou and J. Piekarewicz, Phys. Rev. C60 (1999) 024306.

[22] W. M. Alberico, A. De Pace, G. Garbarino and A. Ramos Phys. Rev. C61 (2000) 044314.

[23] W. M. Alberico and G. Garbarino, Phys. Rep. 369 (2002) 1.

[24] J. Nieves, E. Oset and C. García-Recio, Nucl. Phys. A554 (1993) 509.

[25] J. Nieves, E. Oset and C. García-Recio, Nucl. Phys. A554 (1993) 554.

[26] W. Weise, Nucl. Phys. A690 (2001) 98.

[27] C. García-Recio, J. Nieves and E. Oset, Phys. Lett. B541 (2002) 64.

[28] E. E. Kolomeitsev, N. Kaiser and W. Weise, nucl-th/0207090.

[29] A.Galindo and P. Pascual, Quantum Mechanics, Springer, 1991.

[30] P. Dluzewski et al., Nucl. Phys. A484 (1988) 520.

[31] T. Hasegawa et al., Phys. Rev. C53 (1996) 1210.

[32] J.E. Amaro, J.A. Caballero, E. Moya de Guerra, T.W. Donnelly, A. M. Lallena and J.M. Udias, Nucl. Phys. A602 (1996) 263.

[33] J. Caro, C. García-Recio and J. Nieves, Nucl. Phys. A646 (1999) 299.

[34] J.E. Amaro, C. García-Recio and A.M. Lallena, Nucl. Phys. A567 (1994) 701.

[35] J.E. Amaro, A.M. Lallena and J. Nieves, Nucl. Phys. A623 (1997) 529.

[36] R.B. Firestone, Table of Isotopes ( ${ }^{\text {th }}$ Edition) , John Wiley \& Sons, 1996.

[37] K. Itonaga, T. Ueda and T. Motoba, Nucl. Phys. A691 (2001) 197.

[38] C. Albertus, J.E. Amaro and J. Nieves, Phys. Rev. Lett. 89 (2002) 032501. 